\title{
Erratum to: SISMES VII NATIONAL CONGRESS
}

\section{Padova 2-4 October 2015}

Published online: 16 September 2015

(C) Springer-Verlag Italia 2015

\section{Erratum to: Sport Sci Health \\ DOI 10.1007/s11332-015-0234-0}

The title of abstract 198 SB P contains a typographical error. The correct title is:

"In vivo" flexibility of ski-boots: the effect of boot hinges and ankle joint centre alignment on flex curves.

The title of abstract $\mathbf{3 5}$ SB $\mathbf{K}$ contains a typographical error. The correct title is:

3D body centre of mass trajectory: different computational methods for the same result?

The correct classification of abstract 156 "Altruistic physical activity and city sustainability: a policy-making perspective" is 156 SL K.

The correct classification of abstract 221 "Relationship between Wingate test and vertical jump in young female volleyball players" is 89 TP P.

A new abstract should be added.

\section{0bis AP O}

Adapted Physical Activities (APA) for the mental distress: determinant factors for standardizing intervention procedures

M. Aresti

Department of Human Sciences, Society and Health, University of Cassino and Southern Lazio, Cassino, Italy

Aim: To analyze the impact of a multifactor APA program named "Export-Sport" on the Recovery Process and social behavior of clinically stable schizophrenic patients in order to identify possible guidelines for an APA protocol applied to mental distress.
Methods: 20 patients have undergone a longitudinal intervention study ( 24 weeks) and were divided in an experimental group (EG) (8 males, 2 females, mean age 33.4) and a control group (CG) (5 males, 5 females, mean age 34.5 ). The EG subjects took part in a threetimes-a-week APA program (sport based activities, bioenergeticsoriented expressive activities and swimming activities) and twice a month hiking and nutrition psychoeducation meetings. The CG patients engaged only in body-expression activities twice a week. The pre- and post-intervention tests concerned the amount and type of physical activity (measured through digital 3D accelerometer "Polar Loop Activity Tracker" + "Weekly Diary"), sociality (Moreno's sociogram), life quality and social functioning (QOL), capacity to cope with and overcome difficult situations (Wagnild and Young RS), Recovery Process perception (Recovery Assessment Scale) and levels of needs satisfaction (questionnaire).

Results: The Principal Component Analysis revealed in the EG significantly higher levels of personal activation and positive expectations within the group. The RS confirmed an improvement in coping strategies and problem-solving abilities, with a concomitant increase in the satisfaction levels for health care services. Different life quality items showed a positive trend in both the CG and the EG. Conclusions: The APA intervention should: (1) be devised in close cooperation with a clinical team; (2) include at least two activities systematically distributed during the week; (3) have measurable objectives related to each personal therapeutic and rehabilitative path. Reference

Strassnig M, Signorile J, Gonzalez C, Harvey PD (2014) Physical performance and disability in schizophrenia. Schizophr Res Cognit $1: 112-121$.
The online version of the original article can be found under doi:10.1007/s11332-015-0234-0. 